\title{
Driving assessment with computer-video scenarios: More is sometimes better
}

\author{
WILLIAM SCHIFF, WENDY ARNONE, and STEVE CROSS \\ New York University, New York, New York
}

\begin{abstract}
Several factors are critical in designing effective simulator systems for driving research and driver assessment. First, there is cost, in terms of which, less is better. Costly systems are sometimes fine for specific research purposes, but they are impractical for most other purposes. Second, there is realism; in this case, more may be better, but we don't know how much realism is really necessary for good prediction of actual driving behaviors. Recent evidence suggests that real-world clutter may enhance prediction, but it also makes interpreting research more difficult. More realism may increase cost, but this is not necessarily the case. Third, there is user-friendliness, in terms of which, again, more is better. Systems that are easier to operate require less-highly trained personnel; hence, increasing user-friendliness also decreases cost, especially for assessment. We demonstrated a highly user-friendly, low-cost, moderately realistic, and partly interactive computer-video driving research and assessment system. In recent research, we obtained multiple $R$ values of .47 for older drivers with only three to four scenarios predicting a real-world crash index.
\end{abstract}

Psychologists and others attempting to exploit the recent and rapid development of inexpensive and flexible computer-video systems as means of simulating driving performance have employed several approaches. All of these approaches make assumptions about how best to assess driving. We maintain that these assumptions have constrained the nature of resulting assessment systems, with the systems often being too expensive or complex, or with displays being too abstract to be optimally useful (Schiff \& Arnone, 1994, in press).

\section{Display Realism}

One such assumption concerns display realism. Here, some of us have assumed that more is better. That is, we have assumed that in order to validly tap driving skills, as well as shortcomings or lapses in these skills, we need to present drivers with visual scenes very much like those encountered while driving. If graphics displays are used, they must look very much like real views of road and traffic as seen by the driver. But with this assumption, film or video scenes of driving should be better than graphics. That is, we try to simulate the way things look as closely as possible. Cost considerations may force us to tolerate restrictions in the field of view, optical noninteractivity, or other deviations from complete realism, but in terms of cost, the less the better. We likely absorbed this assumption of the importance of display realism from the field of simulated flying, where cost is relatively unimportant (see, e.g., Warren \& Wertheim, 1990), and from the ecological approach to perceiving and acting (Gibson, 1979), which holds that ecological validity is critical if

Correspondence should be addressed to W. Schiff, Department of Applied Psychology, New York University, 239 Greene Street, Room 537, New York, NY 10003. we are to tap those real-world processes that we are trying to model and that we would like to predict.

Surprisingly little hard evidence is available as to how much realism is enough, but we have a good idea of how much is cost effective. As a result, for the system we have been evolving over the past couple of years, we have used video displays made from tapes shot while actually driving, to assure maximum realism. As far as our participants have mentioned, the displays are reasonably realistic, although more realism would be even better. Clearly, the clientele being assessed considers display realism of paramount importance if the assessment is to reflect the status of its driving skills. In the eye and mind of the person on the street, validity is mainly face validity. Thus, these attitudes are quite important if we are interested in public acceptance of our manner of assessment, and of the advice and counseling that may result from such assessmentof, for example, older drivers (see, e.g., Schiff \& Arnone, 1994; Schiff \& Oldak, 1993).

\section{Response-System Realism}

A second major assumption concerns response-system realism. That is, in order to obtain valid data concerning people's driving from computer assessments or computervideo assessments, the ways in which the driver responds to events in the driving scenario must be highly similar to those in real-world driving. Here, simulators clearly do the best job, since they can mimic real auto controls, yet neither cost nor bulk are especially an issue. However, in our research, and in the design requirements of the system we are trying to develop, both cost and bulk are highly constrained. Therefore, we used commercially available and inexpensive controls, which are similar, although not identical, to actual automobile controls. Interactivity also comes into play here, since for complete real- 
ism, when the driver accelerates, steps on the brake, or turns the steering wheel, the visual display must change accordingly. With video displays such as those we use, while interactivity for accelerator and brake pedals is clearly feasible, for steering, full interactivity becomes impractical due to the number of possible video/film alternatives. Alas, while more control realism may be better, costs and space force us to compromise. Again, where the critical cut-off point is here, we do not know.

We have learned that older drivers in particular do not readily accept or like joystick controls. Further, since hands and arms may be quite different from legs and feet in their response characteristics, we have assumed that it is better to hold the realism course for accelerator and brake pedals, and, more recently, for a steering wheel. Note that we brake via leg extension, but pull back with the hand on a joystick to slow our forward progress. Thus, stimulus-response compatibility may be an issue in translating from auto-type controls to joystick controls. Arthritic specificity and lower-limb impairment via circulatory problems are other issues for older drivers. We would be very cautious about generalizing from hand to foot controls, or vice versa. The ergonomics of our system are not exactly like those of real automobile controls. Our preliminary research project, funded by the AAA Foundation for Traffic Safety, still yielded data that were useful regarding assessment of older and younger drivers, and that included two instances of "unintended acceleration" from our sample of 108 drivers over 55, and none from the 62 drivers under 55 years (see, e.g., Schiff \& Arnone, 1994, Schiff \& Oldak, 1993). Had we obtained these responses with a joystick, their meaning would have been highly questionable.

\section{What to Assess?}

Finally, there is the issue of what to assess. An assumption of many researchers and assessors in the field of driver assessment-especially but not exclusively older driver assessment-is that one should assess dimensions or abilities underlying driving performance. Specifically, they assume that older drivers may be declining in their driving performance because of declines in underlying sensory, perceptual, cognitive, or motor processes. Although we share part of this belief, we do not necessarily accept the corollary assumption that the best way to assess driving performance is to tap the underlying process with abstract psychological tasks, such as those involving contrast-sensitivity thresholds, distance perception, or attention allocation. We assume instead that the best assessment is with driving tasks or situations in which drivers may manifest underlying capabilities in varying degrees. We suggest that since (a) drivers may compensate more or less well for sensory, perceptual, cognitive, and motor declines, and (b) underlying process performances must still be calibrated with actual driving performance, the intermediate examination of underlying process could be skipped. For example, we doubt that an elevated motion-detection threshold or restricted visual field is uniquely linked to high crash risk. Thus these declines still have to be calibrated with actual driving performance if we are to do better than screening out obvious high-risk individuals. Further, because there are only theories, not facts, about the physiological and/or psychological processes underlying driving behaviors, we believe it may be premature to go with the theory, when the practical demands for improved assessment are so pressing.

We also break with some of the tradition in the field of driving assessment by assuming that most of the underlying processes of driving do not include fine static focal resolution (as tapped by static visual acuity measures), but rather involve the timely detection of sets of vectors of rather large objects (e.g., trucks, cars, people), as well as of the signs, signals, and other clues portending vector alteration. We also assume that the underlying processes include the necessary skills and abilities to respond appropriately and in a timely fashion to this higher-order information. It is with all these assumptions in mind, at least most of the time, that the following system components were designed. Specific details are available elsewhere (see Schiff \& Oldak, 1993).

1. User-friendly interface. The system permits automatic or manual presentation and custom scenario presentation-order capability. We also developed a graphic userfriendly readout of results. These components are essential for assessment, while unimportant for research. All instructions for drivers are recorded verbally on videodisc, or may be stored in the computer. They are automatically presented unless aborted by the assessor.

2. Simple RT task. This task comprised five trials involving starting with the gas pedal and then using the braking pedal upon seeing a (graphic) traffic light turn from green to red. It also served as practice with brake and accelerator pedals. We found performance (of 170 drivers aged 15-91 years) to be uncorrelated with crash risk.

3. Preferred driving speed. This task involved four fully interactive speed-selection scenarios in which drivers used the gas pedal and brake pedal to drive at a speed they found comfortable. The scenarios were: no traffic and dry pavement, heavy rain and moderate traffic, light traffic but fog and melting snow on the road, and heavy four-lane highway traffic with oncoming headlight glare. We found older drivers drove more slowly than younger drivers, but especially so when faced with headlight glare. Men drove faster than women, and results paralleled realworld driving habits, as indicated by self-reports. Individual driving records appeared useful for counseling drivers regarding their habitual driving speeds.

4. Hazard detection scenarios. We originally prepared and tested 9 scenarios requiring drivers to detect and respond to events in traffic by braking or steering responses. These were timed from what we considered to be "critical driving incidents" (see Schiff \& Arnone, 1994) contained in these driving sequences. The starts of these incidents (e.g., a pedestrian emerging from between parked cars to step in front of the driver's vehicle) were recorded on 
the computer from inaudible tones inserted in the videodisc scenarios, and processed via a custom tone decoder. A braking response or no response was recorded, with timing accuracy for the entire system of $4 \mathrm{msec}$. Some scenarios were produced in brightness-reduced versions, using videotoaster technology, to serve as functional vision tests in driving situations. Of the 9 scenarios tested, only a few were found to predict crash risk, some for all drivers, and some for drivers over or under 65 years. These results are reported in detail elsewhere (Schiff \& Arnone, 1994), but resulting multiple $R$ values predicting a risk index comprised primarily of real-world crashes were .47 for older drivers and .40 for younger drivers. We are currently testing additional scenarios involving left-turn decisions, steering drifts and timing, and signsignal detection in various traffic/weather conditions.

\section{System Specifications}

Computer. The system, Easy Driver, is an assessment system designed to evaluate abilities related to driving. This assessment system runs on any recent (ca. 1990) Macintosh desktop or notebook computer with two serial ports. Minimal requirements include an internal hard drive and $5 \mathrm{MB}$ of RAM (8 MB is suggested). It is also recommended that, to assist personnel in assessment administration, the computer monitor be at least 12 in., though this is not essential.

MIDI interface and pedals. Two continuous controller MIDI pedals are used to register drivers' responses to materials displayed on a video monitor. The MIDI interface allows the information from the pedals to be input into the computer. The time base of the MIDI standard allows for timing accuracy to $1 \mathrm{msec}$.

Phase locked-loop circuit. A custom electronic circuit was designed to detect the presence of $1-\mathrm{kHz}$ audio tones. It is used to synchronize events on laserdisc with pedal events, allowing both to use the same time base.

Laserdisc player and laserdisc. A Pioneer LD-V8000 computer-controllable laserdisc player is used to display driving scenario materials on any video monitor. A 30-in. or larger high-resolution monitor is recommended. Internal computer storage of video sequences will soon be economically feasible.

Easy Driver program. The program is a custom application that directs the display of driving scenarios via laserdisc player and collects, analyzes, summarizes, and stores input from the pedals or other input device (e.g., steering wheel). Data may be displayed graphically, with danger zones indicated by color/texture coding.

Steering-wheel system. The steering wheel (Colorado Spectrum Mouse-Wheel) is used for left-turn and hazardsituation assessment by tracking the mouse position timed in relation to audio timing tones. The Easy Driver system obtains the mouse position and tracks the position as determined by the driver's turning of the steering wheel. The degree to which the wheel is turned and when it is turned may also be obtained.

Mountings. Pedals are mounted on a hinged wooden base providing pedal spacing and angles similar to those found on most passenger cars. Since the driver's chair rests on the base, it is horizontally secure. The steering wheel is mounted on a simple wooden platform that again provides reasonable realism.

\section{REFERENCES}

GiBson, J. J. (1979). The ecological approach to visual perception. Boston: Houghton Mifflin.

SCHIFF, W., \& ARNONE, W. (1994). Assessing older drivers with critical driving incidents. Unpublished manuscript.

SCHIFF, W., \& ARNONE, W. (in press). Perception and driving: Where parallel roads meet. In J. Flach, P. Hancock, J. Caird, \& K. Vincente (Eds.), Global perspectives on the ecology of human-machine systems. Hillsdale, NJ: Erlbaum.

SCHIFF, W., \& OLDAK, R. (1993). Functional screening of older drivers using interactive computer-video scenarios. Washington, DC: AAA Foundation for Traffic Safety.

Warren, R., \& Wertheim, A. H. (EDs.) (1990). Perception and control of self-motion. Hillsdale, NJ: Erlbaum. 